\title{
Reflets
}

Revue ontaroise d'intervention sociale et communautaire

\section{" Les chemins qu'on parcourt pour livrer un combat contre la maladie sont très difficiles par bouts "}

\section{La réalité des Franco-Manitobaines et le cancer du sein}

\section{Elsy Gagné}

Volume 1, numéro 2, automne 1995

La santé communautaire en Ontario français : défis et espoirs

URI : https://id.erudit.org/iderudit/026078ar

DOI : https://doi.org/10.7202/026078ar

Aller au sommaire du numéro

Éditeur(s)

Reflets : Revue ontaroise d'intervention sociale et communautaire

ISSN

1203-4576 (imprimé)

1712-8498 (numérique)

Découvrir la revue

Citer cet article

Gagné, E. (1995). « Les chemins qu’on parcourt pour livrer un combat contre la maladie sont très difficiles par bouts " : la réalité des Franco-Manitobaines et le cancer du sein. Reflets, 1(2), 90-114. https://doi.org/10.7202/026078ar

\section{Résumé de l'article}

Suite à une recherche qualitative effectuée auprès de 20 femmes vivant en français au Manitoba,cet article fait état de leur expérience avec le cancer du sein et du rôle actif des groupes desoutien. Il s'agit de comprendre plus profondément comment les femmes francophones vivantau Manitoba ont réagi à et vivent avec la maladie et de présenter les initiatives développéespar et pour les femmes pour leur venir en aide afin de contrer l'isolement et le rejet social.Avec le temps, on verra que la maladie devient une manière d'être et de vivre, une occasionde dire et de faire le sens de sa vie et de sa mort. C'est grâce à cette expérience que ces femmespartagent un sentiment

d'appartenance à une communauté basée sur la langue d'usage, lamaladie et la survie et qu'elles expriment le besoin de se reconnaître en tant que groupe et departager un héritage culturel et une expérience sociale bien définis.
Tous droits réservés (C) Reflets : Revue ontaroise d'intervention sociale et communautaire, 1995
Ce document est protégé par la loi sur le droit d'auteur. L'utilisation des services d’Érudit (y compris la reproduction) est assujettie à sa politique d'utilisation que vous pouvez consulter en ligne.

https://apropos.erudit.org/fr/usagers/politique-dutilisation/ 


\section{"Les chemins qu'on parcourt pour livrer un combat contre la maladie sont très difficiles par bouts»}

\section{La réalité des Franco-Manitobaines et le cancer du sein}

Suite à une recherche qualitative effectuée auprès de 20 femmes vivant en français au M anitoba, cet artide fait état de leur expérience avec le cancer du sein et du rôle actif des groupes de soutien. II s'agit de comprendre plus profondément comment les femmes francophones vivant au $M$ anitoba ont réagi à et vivent avec la maladie et de présenter les initiatives développées par et pour les femmes pour leur venir en aide afin de contrer l'isolement et le rejet social. A vec le temps, on verra que la maladie devient une manière d' être et de vivre, une occasion de dire et de faire le sens de sa vie et de sa mort. $C$ 'est grâce à cette expérience que ces femmes partagent un sentiment d'appartenance à une communauté basée sur la langue d'usage, la maladie et la survie et qu' elles expriment le besoin de se reconnaître en tant que groupe et de partager un héritage aulturel et une expérience sodale bien définis.

\section{E lsy $\mathbf{G}$ agné}

C hercheure indépendante, $C$ ollège universitaire de St-B oniface, $M$ anitoba

II n'est pas facile de résumer les initiatives développées par et pour les femmes franco-manitobaines vivant ou ayant vécu avec le cancer du sein et les préoccupations de ces femmes. Les enjeux sont très diversifiés et la culture ou sous-culture au coeur de l'expérience de la maladie varie en fonction des provinces, des régions, des milieux, des communautés d'appartenance, des groupes, des valeurs culturelles et, bien sûr, de la gravité de la maladie. La maladie n'est plus ce qu'elle était, ayant changé de forme grâce à 
la médecine moderne. Selon les médecins et les oncologistes, il est aujourd'hui possible de lutter contre le cancer du sein.

Cet article vise une meilleure connaissance de la réalité sociale des femmes franco-manitobaines qui vivent ou ont vécu avec le cancer du sein, par un regard sur les initiatives développées à l'intention de celles qui vivent en français dans leur milieu d'origine. O n notera qu'au C anada, le cancer du sein demeure la deuxième cause de mortalité. Au M anitoba seulement, on observe un nombre de 730 nouveaux cas sur les 17700 nouveaux cas au C anada (Statistique C anada, 1995), ce qui souligne l'importance de cette maladie et ses effets dans la vie quotidienne des Franco-M anitobaines et de leur famille immédiate.

D ans cet essai, je m'arrêterai d'abord à montrer sur quoi portent les recherches sur l'expérience de la maladie et l'influence prépondérante de la culture ou sous culture dans l'expérience humaine, comme c'est le cas de l'expérience de la maladie. Ensuite, je montrerai comment la méthodologie qualitative reflète une approche d'analyse particulière qui tient compte du vécu individuel des femmes franco-manitobaines et de leur rapport avec le social et le médical. Je présenterai enfin l'analyse du dis cours des femmes franco-manitobaines qui vivent ou ont vécu avec le cancer du sein en me servant des réseaux sémantiques comme moyen de regrouper l'information recueillie et de rendre compte de la problématique étudiée.

\section{Cadre théorique}

Plusieurs études (Herzlich, 1984; Good et DelVecchio-Good, 1981; Good, 1977) ont mis en relation maladie et malaise en sappuyant sur le concept de culture. Ces recherches s'appuient sur l'idée qu'il existe dans chaque culture un ensemble de symboles dont le pouvoir et la charge symbolique sont enseignés et partagés par l'ensemble des membres de la communauté. Good (1977), qui a décrit la sémantique de la mal adie du coeur en I ran, 
a découvert comment celle-ci est associée culturellement à une grande variété de situations, d'états affectifs et de facteurs stressants. A utrement dit, le sens de la maladie est relié à un syndrome d'expériences typiques, à un ensemble de mots, d'idiomes et de sensations qui sont regroupés et forment entre eux des réseaux sémantiques. $O r$, c'est grâce à l'analyse du langage d'individus qui vivent l'expérience de la maladie qu'on peut saisir les réseaux sémantiques. Ainsi, ceux-ci traduisent un ensemble de valeurs, de signes et de symboles propre à la culture ou sous-culture des malades. Kleinman (1986) appuie également cette thèse. Dans une étude où il a documenté le processus de transaction entre malades et thérapeutes à propos de la neurasthénie et de la dépression en Chine, Kleinman (1986) considère le diagnostic médical comme un processus à caractère essentiellement sémiotique. L'auteur précise que poser un diagnostic signifie donner un sens socialement construit à la maladie. D u point de vue du malade, ce sens social peut être considéré comme l'une des valeurs possibles que peut prendre la maladie.

Par culture, il faut entendre «cette totalité où entrent les ustensiles et les biens de consommation, les chartes organiques réglant divers groupements sociaux, lesidées et les arts, les croyances et les coutumes» (Van M etter, 1992:443). Elle est un vaste appareil matériel, humain ou spirituel qui aide l'individu à affronter les problèmes qui se posent à lui. C'est en ces termes que Saillant (1988) s'intéresse à la culture et à son influence dans le déroulement de la maladie. R eprenant à son compte l'analyse de Foucault (1972), l'auteure explique que la maladie est à la fois une construction historique et culturelle. Pour que cela soit, de véritables dispositifs sociaux sont manifestement à l'oeuvre. Par dispositifs, il faut entendre les discours, les institutions, lesénoncés scientifiques, la moral ité et les propositions philosophiques entourant la maladie. $O r$, en abordant la question de la maladie et de la culture au sens historique de Foucault, Saillant (1988) affirme que «a culture est au centre de toute expérience humaine, incluant bien sûr, l'expérience de la maladie» (p.13). En ce sens, elle permet sa création historique. Ainsi, l'expérience du cancer est une réalité qu'il nous est possible de mieux comprendre en la restituant dans 
«A insi, l'expérience du cancer est une réalité qu'il nous est possible de mieux comprendre en la restituant dans un contexte d' analyse plus global des interactions, des organisations, des rites culturels et religieux, et des symboles. Tout cela permet de définir la maladie, de la rendre possiblement vivable et aussi de la vaincre. » un contexte d'analyse plus global des interactions, des organisations, des rites culturels et religieux, et des symboles. Tout cela permet de définir la maladie, de la rendre possiblement vivable et aussi de la vaincre.

Dans son étude, Saillant (1988) affirme que la maladie est plus qu'un corps de symptômes qui renvoie essentiellement à un monde biologique. Elle est entendue comme «un mode de vie, un acte de communication, une occasion de dire et de faire le sens de sa vie et de sa mort» (p.13). D'où l'idée selon laquelle il est possible de vivre avec le cancer et que ce vécu varie selon plusieurs facteurs dont le milieu d'appartenance et l'origine ethnique. Plusieurs analystes (Good et DelVecchio-Good, 1981, 1982) ont, à leur tour, montré que la présentation des symptômes varie d'un groupe ethnique à un autre à cause de la culture dont ils sont porteurs. Good et DelVecchio-Good (1981) s'entendent pour reconnaître que les normes et les valeurs du milieu ou du groupe d'appartenance sont reliéesà un ensemble de significations particulières. C elles-ci se comprennent davantage dans l'expérience de la maladie, telle qu'enracinée au coeur du quotidien et de la culture.

D'après D elVecchio-Good (1993), et dans ses tentatives anthropologiques d'étude du quotidien en clinique américaine et japonaise, lesoncologistes américains ont reçu le mandat culturel d'instiller l'espoir dans les récits thérapeutiques qu'ils façonnent avec leurs patients et leurs patientes en tentant d'éveiller chez eux un sensdu temps présent plutôt qu'une perception purement chronologique du temps. 0 r, dans ses tentatives anthropologiques d'étude du quotidien en cliniques américaines et japonaises, D elVecchio-Good (1993) précise que les notions contrastantes de temps et de dévoilement de l'information font ressortir les différences culturelles entre les groupes ethniques. Celles-ci se manifestent par la diversité des conceptions reliées à la spécificité des horizons temporels, aux luttes, voire aux idéaux culturels présents dans la formulation des dénouements. D'un côté, les oncologistes japonais révèlent rarement le diagnostic de cancer; ce dernier révélé, le patient ou la patiente doit s'attendre à ce que 
«... dès le diagnostic connu et la chirurgie exécutée, la vie de femme se brise et se déconstruit pour ensuite se rebâtir à nouveau.» sa maladie soit fatale. Les proches sont mis au courant de la nature de la maladie au cours des consultations antérieures. D e leur côté, les oncologistes américains divulguent l'information directement à leurs patients et à leurs patientes dans le but précis de les faire participer au processus thérapeutique, créant ainsi un climat social de confiance propice à l'échange, si tel est leur désir, pendant leurs traitements médicaux.

Wong (1995) est d'avis que le meilleur traitement médical pour contrôler le cancer du sein demeure la mastectomie, c'està- dire l'ablation du sein. De cette opération chirurgicale découlent des effets psychologiques (Schain, 1991) qui ont été observés. O n pense ici à la dépression, la mauvaise estime de soi, I'anxiété et le sens diminué de la féminité (Torrie, 1971). Selon Gauthier (cité par Bouchard, 1995), qui tente d'expliquer les effets psychologiques, dès le diagnostic connu et la chirurgie exécutée, la vie de la femme se brise et se déconstruit pour ensuite se rebâtir à nouveau. C ette brisure se comprend davantage par l'agression corporelle causée par la chirurgie, où la femme qui veut être sauvée (Bégin, 1989) perd une partie d'elle-même. À la suite de la chirurgie, la femme vit également une expérience-choc (Schutz, 1987) qui l'incite à faire éclater le sens de sa vie et lui permet de modifier son comportement. C'est grâce à l'usage de la prothèse ou la reconstruction du sein que l'ajustement s'effectue.

Suite à l'ablation d'un sein, la femme est durement marquée par la perte d'un membre et par une cicatrice physique. Dans leur étude, D orvill, R enaud et Bouchard (1994) soulignent que la caractéristique de l'asymétrie du corps différent de la norme suscite une déstabilisation. L'imperfection du corps signifie un «chaos dans l'ordre de la pensée humaine, la représentation sociale du handicap» (p.718). Le handicap devient dèslorsune nouvelle for me de stigmatisation (G offman, 1975). C ar pour la femme qui a subi une mastectomie et survécu au cancer du sein, son corps n'incarne plus les formes pures, la perfection et la beauté de la femme.

D ans l'ensemble, on comprend bien que toute expérience du cancer n'est pas nécessairement reliée à la mort. Les analystes 
«D ans leurs efforts, les groupes de soutien redonnent l'espoir aux malades et leur offrent des moyens de reprendre leur vie en main (confiance en soi et prise de pouvoir).»
(Saillant, 1988; Gauthier, 1995) sont d'avis qu'on peut vivre pendant et après le cancer. C'est grâce au rôle joué par les groupes de soutien qu'il est possible d'appuyer les malades ayant traversé l'expérience du cancer dans leur milieu de vie social et naturel. $D$ ans leurs efforts, les groupes de soutien redonnent l'espoir aux malades et leur offrent des moyens de reprendre leur vie en main (confiance en soi et prise de pouvoir). D es analystes font référence à un soutien familial parce que la famille devient le premier et ultime recours (Fortin, 1994). Ainsi, on dira qu' «avant de recourir aux institutions, on se tourne d'abord vers ses proches, et en tout premier lieu sa famille» (p.947). U n tel recours de la part de la famille permet de se soutenir en période de difficulté ou de crise pour ainsi pallier à l'isolement. À ce réseau familial se joignent également le réseau relationnel et le réseau naturel. M assé (1993) mentionne que le soutien social joue le rôle de tampon, agit comme facteur de protection et permet d'atténuer les conséquences psychologiques et sociales de l'exposition aux situations stressantes, tellesque l'expérience du cancer du sein. L'auteur soutient que l'amélioration de l'état de santé passe par la modification des facteurs environnementaux. Saillant (1988) appuie également cette thèse. Elle précise que les personnes survivant au cancer doivent s'adapter à un nouveau mode de vie, une telle adaptation ayant des conséquences dans leur vie et dans celle de leur famille. S'adapter à son nouveau mode de vie ne signifie pas accepter un diagnostic comme celui du cancer, mais bien de vivre avec la réalité de la maladie et de tout ce qu'elle engendre sur le plan humain (Gauthier, 1995).

\section{Méthodologie}

Afin de mieux comprendre la réalité des Franco- $M$ anitobaines qui vivent ou ont vécu avec la maladie, on a eu recours à une méthode qualitative, soit un exercice structuré de mise en relation logique de variables et de catégories de données. Par ce type 
«... une vingtaine d'entrevues ont été réalisées auprès de femmes âgées de 36 à 79 ans, dispersées pour la plupart dans laVallée de la R ivière $R$ ouge au $M$ anitoba et qui parlent le français, malgré le peu de services médicaux offerts dans leur langue maternelle» d'exercice, on tente de reproduire logiquement un schéma mental de l'expérience du cancer du sein en tant que phénomène. II s'agit par là de vérifier, par les entrevues en profondeur et l'observation, le degré de correspondance entre cette construction ou création de l'esprit et la situation réelle dans laquelle vivent les individus. $C$ 'est donc à partir du discours des individus qu'on tente de reconstituer les modèles culturels et d'expliquer un ensemble d'éléments de références à partir desquels se comprend l'expérience de la maladie. L'individu devient un échantillon de son groupe d'appartenance et c'est à partir desanecdotes, des moindres événements qui émanent de sa quotidienneté et se rapportent à l'expérience sociale du cancer du sein que l'on tente d'appréhender ce qu'il partage avec d'autres. 0 n pense notamment aux valeurs religieuses et à leur encadrement philosophique apporté dans la représentation de la maladie. II y a aussi I'usage répétitif des mots tels que la vie et la mort, le temps et l'espoir, la lutte et les enfants, le corps et la marque. C'est grâce au découpage des mots, des idiomes, des signes et des valeurs émis lors des entrevues en profondeur portant sur l'expérience du cancer du sein qu'il a été possible de repérer les réseaux sémantiques des femmesfranco-manitobaines. C ela nousaide donc à mieux comprendre le contenu des récits de vie.

C'est dans un tel contexte qu'il importe, selon Bertaux (1976), de considérer les récits de vie comme pratiques culturelles et d'éviter de centrer uniquement notre réflexion sur une vie. $C$ ar les récits de vie (Grell, 1986; H oule, 1986) et l'analyse du discours apparaissent comme un moyen de saisir la réalité vécue par les femmes franco-manitobaines et qui, à la rigueur, se distingue du vécu des autres femmes étant donné le milieu d'origine et la situation minoritaire francophone. La collecte des données révèle une attitude nouvelle: approcher l'histoire d'individus par leur petite histoire, celle qui se joue au quotidien et celle d'individus que l'on pense sans histoire (Le G all, 1987). C'est l'échantillonnage boule de neige qui a rendu possibles ces récits de vie. Et pour la recherche récente, et encore inachevée, une vingtaine d'entrevues ont été réalisées auprès de femmes âgées de 36 à 79 ans, dispersées pour la plupart dans laVallée de la R ivière $R$ ouge 
au $M$ anitoba et qui parlent le français, malgré le peu de services médicaux offerts dans leur langue maternelle. Pour la plupart d'entre elles, I'opération chirurgicale s'est effectuée à l'H ôpital Général de Saint-Boniface. Le choix des sujets rencontrés porte essentiellement sur la définition suivante: ce sont des femmes franco-manitobaines vivant en français, ayant eu le cancer du sein et subi une mastectomie.

D ansl'ensemble, les entrevues en profondeur portent sur plusieurs aspects, telsl'expérience de la maladie à partir des premiers symptômes et du diagnostic médical, des types de réactions face à la nouvelle, des traitements pour contrôler la tumeur, du réajustement face à son nouveau mode de vie et du rôle de la culture religieuse ainsi que sa signification. D'autres questions mettent en évidence les initiatives développées par rapport aux groupes de soutien (français et anglais), le retour possible à la santé, la volonté de vaincre son cancer, le temps, l'identité féminine et les perceptions par rapport à son corps.

\section{Les résultats}

Pour des fins pratiques, je présenterai les questions pertinentes à la problématique ici étudiée, à savoir l'expérience de la maladie vécue par les femmes franco-manitobaines et les initiatives développées dans leur communauté d'appartenance en milieu rural. A fin de mieux repérer les systèmes sémantiques, l'accent sera mis sur les thèmes porteurs de sens tels que la terrible nouvelle, le corps, la maladie, la vie, l'espoir, le refus de la mort, la foi en Dieu, les enfants et la famille.

\section{Expérience sociale du cancer du sein}

Le point de vue des femmes franco-manitobaines a révélé des éléments clefs sur la manière dont elles s'y prennent pour traverser l'expérience sociale du cancer du sein. Aux questions: Comment avez-vous réagi au diagnostic comme celui du cancer 
«L orsqu'il pose le diagnostic, c'est comme si tout s' écroulait autour de toi, il n'y a plus de sens à ta vie. L a maladie devient le centre de tes adivités... » du sein? Comment vivez-vous ou avez-vous vécu l'expérience de la maladie et quels sont les moyens qui aident à traverser cette expérience?, plusieurs réponses permettent de mieux articuler l'analyse du changement individuel, familial et social vécu par les femmes rencontrées, changement provoqué par la venue de la maladie et confirmé officiellement par le diagnostic médical.

Soulignons le rôle crucial joué par les médecins dans les rencontres médecin/ patiente, notamment lors de la divulgation de la terrible nouvelle. $D$ ans la relation médecin/ malade, on constate que les oncologistes canadiens, dont font état les répondantes, divulguent l'information à leurs patientes en les invitant à participer activement au processus thérapeutique, avec pour effet de créer un climat de confiance entre les deux parties et d'instiller l'espoir dans les récits thérapeutiques (D elVecchio-G ood, 1993). Selon les témoignages des patientes, les oncologistes canadiens semblent développer chez elles un sens du moment présent, c'està-dire de l'immédiateté. II existe là un contraste réel entre le dévoilement de la terrible nouvelle (rapport médical qui se rapporte à son état de santé) et l'éveil du moment (temps) présent. U $n$ tel contraste se mesure en effet dans le discours des femmes franco-manitobaines.

«D u point de vue psychologique, vivre l'expérience du cancer du sein t'amène à l'âge de 60 ans, si tu en as seulement 45 ans (...). Tout arrive très rapidement. Tu localises une petite bosse sur un sein et le médean te confirme la terrible nouvelle. L orsqu'il pose le diagnostic, c'est comme si tout s'écroulait autour de toi, il n'y a plus de sens à ta vie. La maladie devient le centre de tes activités (... ). Je me disais que cela n'était pas possible que je sois réellement rendue à ce point-là dans ma vie (... ). E nsuite, tu te retrouves à I'hôpital et tu deviens une patiente comme les autres. Tu sens un corps malade (... ). M ais rapidement j'ai accepté d'avoir la mastedomie pour être encore avec mes enfants, ma lueur d' espoir! M ais, pendant tout ce temps (... ), on pense beaucoup plus à la mort (... ). 
J'ai eu une dépression et mon estime de soi en a pris un coup. E $t$ plus tard, l'acceptation fait partie de ton cheminement psychologique (... ). Le médean $\mathrm{m}$ 'a donné de l'espoir et le courage de me battre $C$ 'est vraiment grâce à l'espoir et à ma foi quej'ai pu m'accrocher à la vie (... ). D eux ans après la mastectomie, j'ai eu la reconstruction du sein (... ). L e moment passé avec la maladie m'a beaucoup fait réfléchir (... ). Je ne vois plus les choses de la même manière J'apprends à penser un peu plus à moi (... ). J'ai appris à vivre au jour le jour.»

C ette jeune femme, née au $Q$ uébec et vivant au $M$ anitoba depuis 20 ans, a précisé que sa mère est morte du cancer du sein en 1956, ce qui engendre dans sa vie de fille ainsi que dans celle de ses frères et soeurs, un déséquilibre psychologique et social qui marquera sa vie. Son père, devenu dès lors veuf, prend la décision de placer ses enfants dans des foyers d'accueil afin de leur garantir une meilleure éducation par des femmes. C e déménagement entraînera chez elle un réajustement et générera un sentiment d'insécurité de se savoir à nouveau délaissée. Lors de l'expérience du cancer du sein, la répondante a revécu un sentiment d'insécurité et la peur de laisser elle aussi ses trois enfants.

«) voulais donner à mes enfants une stabilité et ne pas les faire vivre ce quej' ai vécu à leur âge lorsque ma mère est morte Je m'accrochais à la vie. Je voulais prendre toutes les chances de mon côté. Je voulais me battre pour mes enfants et aussi pour moi-même»

D ans le même ordre d'idées, une autre patiente met l'accent sur ce besoin de redevenir la femme d'avant, en santé, pour ainsi reprendre la routine normaleet d'accepter sa condition de femme. O n dira ici que la santé se mesure à partir d'une capacité d'agir dans son milieu et d'accomplir les tâches ménagères et sociales.

«Tout s'est passé tellement vite dans mon cas $E n$ moins de dix jours, j'ai appris la nouvelle et j'ai eu 
« $\mathrm{n}$ dira donc que survivre au cancer du sein implique une double acceptation: celle d'avoir l'opération et celle de vivre avec la réalité de la maladie et ses marques corporelles... » mon opération (... ). M on médedn me parlait de lutter contre la maladie et de prendre le temps comme il vient (... ). J'ai donc eu l'opération pour augmenter mes chances de survie (... ). À la suite de mon opération, on m'a amenée à la salle de réveil. Et le premier geste que j'ai fait fut celui de poser la main sur ma cicatrice (... ). J'ai eu peur. Je savais aussi que j'étais sauvée à temps par les médeans (... ). J'ai eu un choc en touchant à mes côtes (... ). J'avais I'impression que j' étais frappée par quelque chose d'horrible II y avait quelque chose que je n'avais plus et surtout que je n'aurai jamais plus $M$ ais en même temps j'étais tellement déterminée. Je voulais revenir à la maison et reprendre ma routine de vie normale (... ). L es mois ont passé et, plus tard, j'ai réalisé que j'aurais pu en mourir. $E t$ là, mes réactions ont été fortes. $0 \mathrm{n}$ aurait dit que je refusais d'être une femme anormale (... ). A ujourd'hui, et malgré que je sois différente des autres femmes et que j'aie encore de la difficulté à accepter ma nouvelle condition de femme, je suis une survivante. Je ne me sens pas malade Je fais œ que je veux dans la maison.»

Être sauvée par la médecine moderne et vivre sa condition de femme en assumant les conséquences physiques et psychologiques de l'opération sont deux composantes importantes à considérer dans l'étude du cancer du sein. O n dira donc que survivre au cancer du sein implique une double acceptation: celle d'avoir l'opération et celle de vivre avec la réalité de la maladie et ses marques corporelles (la cicatrice causée par l'ablation du sein et quelquefois les effets de la radiation). De cette double acceptation découle une nouvelle adaptation à sa condition de femme devenue durement marquée par l'expérience sociale de la maladie. La vie de femme se brise complètement pour se reconstruire à nouveau. $R$ evivre sa vie dans un corps mutilé s'effectue graduellement avec le sens du temps présent. C ela permet aux femmes de se protéger contre l'impondérable. 
«D ans l'expérience du cancer, on peut être sauvée Pour moi, être sauvée, c'est avoir à nouveau la santé. Et c'est ce quej'ai. A ujourd'hui, je peux dire que je fais tout ce que je veux dans la maison. Je fonctionne maintenant avec œe que j'ai. M ais c'est grâce au mé decin que je suis sauvée $E n$ m'annonçant la terrible nouvelle, mon médean $m^{\prime}$ a dit de ne pas $m^{\prime}$ inquiéter et c'est ce que j'ai fait. II n'y avait pas grand chose à faire, tu sais»

«I faut vivre au jour le jour. Si quelque chose va mal, tu penses toujours à la possibilité que revienne le cancer. $\mathrm{Q}$ uoique tu fasses, tu as toujours cette idée qui te revient. $\mathrm{M}$ oi, je pense que l'expérience du cancer fait dorénavant partie de ma vie II faut garder espoir que tout va bien se passer.»

«Tu sais, nous aussi, on est humain. II arrive des moments où tout va bien et on oublie un peu notre situation. 0 n est capable de se détacher de la maladie Parce qu'elle fait partie de notre corps, de notre vie quotidienne, on ne peut pas I'oublier. À chaque fois qu'il $m^{\prime}$ arrive quelque chose, je suis inquiète $M$ on état est là et, pour moi, c'est constant! $M$ ais il faut garder espoir.»

De cela, on retient quatre élémentsimportants: la nécessité d'avoir I'opération pour augmenter ses chances de survie; I'acceptation de vivre avec sa maladie; I'optimisme et l'espoir, et enfin, la confiance que l'on a d'être sauvée dans un avenir plus ou moins rapproché en étant éveillée au sens de l'immédiateté. II se peut que lesconditionsde la maladie vécue par lesFranco- $M$ anitobaines ayant subi l'opération n'aillent pas en s'améliorant. La détérioration de la santé peut s'accompagner d'un désordre psychologique dont nous a déjà parlé Torrie (1971). Ce qui amène la femme atteinte à provoquer des changements immédiats dans sa réalité quotidienne. Voyons comment cela se traduit dans les faits. 
«D epuis ma première opération, en 1984 , je suis toujours malade. Je vais tout le temps à l'hôpital. Je vis seule la douleur. Je ne veux pas montrer à mes enfants que je suis faible et malade $M$ ais je suis très blessée et surtout seule à vivre ma maladie $M$ on mari ne $\mathrm{m}^{\prime} \mathrm{a}$ jamais aidée dans quoi que ce soit dans ma vie A voir le cancer du sein ne signifie pas grand chose pour lui. II me bouscule comme il l'a toujours fait (... ). J'ai besoin d'aide (... ). E t je ne reçois que de l'agressivité et du déchirement psychologique (... ). C e n'est pas très rassurant $d$ ' avoir le cancer du sein, de recevoir des traitements et d'être suivie étroitement par les médeans Tu ne sais jamais si c'est toi qui va y passer la prochaine fois. II y a beaucoup de femmes francophones qui meurent dans notre région à cause du cancer du sein (... ). J'essaie de trouver dans ma vie une lueur d'espoir. Je cherche la tendresse et l'amour. J'évite de plus en plus les problèmes et la confrontation avec mon mari. Je n'ai plus le temps! Je suis toujours malade et souvent à l'hôpital. Je suis toujours inquiète. Et la maladie habite aussi ma vie en silence. Je n'ai pas le choix car en me regardant dans le miroir ou en pre nant ma douche, je vois mon corps marqué, coupé et brûlé par la chirurgie et les effets de la radiation. C 'est terrible, mais que puis-je faire? C'est là pour toujours (...).»

Pendant les semaines qui ont suivi cette entrevue, cette femme a quitté son mari pour vivre avec celui qui lui apporte ce dont elle a besoin pour vivre sa vie quotidienne. $0 \mathrm{n}$ remarque également la question de temps qui revient dans le témoignage proposé ici. La détérioration de la maladie engendre un déséquilibre psychologique, mais aussi un besoin immédiat de réconfort qui se traduit en termes de séurité dans l'insécurité de vivre avec la maladie. Je précise. Cette idée de voir mourir lesfemmesfranco-manitobaines dans la région rurale à cause du cancer du sein revient à quelques reprises lors des entrevues. II y a dans le langage utilisé une peine 
de se voir devenue malade comme les consoeurs et en même temps une peur de mourir pour les mêmes raisons. Ces sentiments (peine et peur) font partie de la réalité des Franco$M$ anitobaines qui vivent ou ont vécu avec le cancer du sein. «Tu ne sais pas si ce ne sera pas toi qui va y passer la prochaine fois» est une phrase qui est souvent utilisée dans le langage des répondantes et qui marque un temps de réflexion dans leur discours.

Et puis, la détérioration physique peut également amener les femmes rencontrées à ne plus espérer survivre à cette terrible maladie qu'est le cancer du sein, comme dans le cas de cette femme qui a survécu pendant près de 10 ans au cancer du sein et qui y a succombé en janvier 1995.

«A près mon expérience avec cette terrible maladie (le cancer du sein) qui a été suivie par un cancer au poumon et un autre au cerveau, j'ai fait une dépression. J'ai été traitée par un psychiatre (... ). Tu saiş, lorsqu' on m'a annoncé que j'avais un cancer du sein, j'ai eu un choc Veux, veux pas, tu peux regarder cela du sens que tu veux, mais tu perds une partie de toi, de ta féminité (... ). J'avais un enfant de quatre ans Je n'étais pas prête à mourir. II était important dans ma vie et je savais qu'il avait besoin de sa mère (... ). Pendant 10 ans, tout s'est bien passé. E t puis, j'ai été à nouveau malade. L e cancer est revenu. A ujourd' hui, c'est encore ce qu' on essaie de combattre. II y a des moments où je me demande vraiment comment tout cela va se dérouler, mais je lutte encore contre la maladie»

Cette patiente témoigne d'abord du choc qui la propulse dans I'univers de la maladie et ensuite du refus de laisser son fils en bas âge. À l'aube de ses trente ans, cette femme subit le choc d'une transformation intérieure dans sa vie. Elle entre dans un monde où son rôle de femme ne sera pas celui qu'il aura été jusqu'ici et en même temps affectera son rôle de mère. En effet, cette femme, aux prises avec le cancer du sein, vivra un changement d'attitude par rapport à la signification de sa vie. À cela śajoute une prise 
«L es groupes de

soutien leur permet-

tent d' affronter la maladie et doivent

être pris en considération lorsqu' on s'intéresse à la santé communautaire des femmes francomanitobaines, tant au niveau individuel que collectif.» de conscience d'un passage possible de la vie à la mort.Vivre l'expérience de la maladie, pour certaines, c'est être consciente d'une mort éventuelle et de la perte de son rôle de mère. Autrement dit, il y a là ce sentiment social de se sentir toujours utile aux yeux des enfants. Et le refus de laisser son fils signifie le refus de la mort, le besoin d'un refuge et l'espoir de survivre au cancer du sein.

Bien que les contextes d'analyse soient différents de ceux présentés par Good (1977), il n'en demeure pas moins que les résultats sont communs ou parallèles. En ce sens la sémantique de la maladie est associée à une variété de situations, d'états affectifs et d'agents stressants. Le sens donné à la maladie du cancer du sein est relié à des expériences typiques telles que la prise en charge de sa vie, les rencontres médecins/ patientes (peu développées ici vu que cette problématique dépasse largement le cadre théorique du présent essai), le déchirement psychologique et social, la volonté de lutter contre la maladie et l'importance de la culture religieuse. Comme nous venons de la constater, il y a en effet un ensemble de mots et d'idiomes lourds de sens dans la vie des femmes. O n pense par exemple à la maladie, à la lutte et à la mort, ou encore, son essaie de trouver dans sa vie une lueur d'espoir». Enfin, une telle analyse permet de montrer comment la culture/ sous-culture franco-manitobaine influence la maladie et détermine le savoir-faire des médecins ainsi que le fonctionnement des groupes de soutien.

\section{Les groupes de soutien}

U ne fois le diagnostic de cancer du sein connu, plusieurs scénarios s'offrent aux patientes pour les aider à reprendre confiance en elles et à prendre le contrôle de leur vie. En effet, «cela va d'amis qui s'éloignent, par malaise ou par crainte de la mort, à I'entourage qui surprotège, sans compter les problèmes de communication» (G authier, 1995:8). Les groupes de soutien leur permettent d'affronter la maladie et doivent être prisen considération lorsqu'on s'intéresse à la santé communautaire des femmes francomanitobaines, tant au niveau individuel que collectif. On verra 
comment cela est effectué par des individus qui se sont définis une communauté d'appartenance en milieu rural. La plupart des témoignages proviennent de Franco- $M$ anitobainesqui ont comme fonction d'amener les femmes vivant ou ayant vécu le cancer du sein à (re)prendre en main leur vie. Le fait pour elles de vivre relativement dispersées a certainement un impact dans leurstentatives de regroupement. C'est dans un tel esprit que les groupes de soutien appuient les femmesfranco-manitobaines rencontrées qui ont subi la mastectomie (traitement médical pour contrôler le cancer du sein) et suivi des traitements chimiothérapiques ou de radiation. $D$ ans l'ensemble, on a constaté que les groupes de soutien peuvent faciliter le passage entre l'état de la maladie et l'état de santé. C'est du moins ce qui se dégage de l'analyse du discours des femmes franco-manitobaines qui ont besoin d'aide pour accepter et vivre avec la réalité de la maladie, réalité souvent difficile à supporter lorsqu'on est seule et isolée.

Le fait de communiquer avec sa famille et ses pairs, d'établir une relation de confiance avec son conjoint pendant et après la maladie, de se savoir soutenue dans une période insoutenable est à considérer lorsqu'on s'intéresse notamment au rôle des groupes de soutien. C eux-ci peuvent offrir des sessions pendant lesquelles les épouses et les maris, par exemple, peuvent échanger sur ce qu'ils vivent dans leur vie quotidienne. La communication entre les Franco-M anitobaines vivant ou ayant vécu la maladie joue un rôle important pour plusieurs d'entre elles.

«II y a des groupes de support à W innipeg, mais pas dans la région de la $R$ ivière $R$ ouge. $P$ our moi, ils sont importants. Je vais te donner un exemple $D$ ans le déroulement de ma maladie, je n' ai jamais vraiment su ce que mon mari ressentait à mon égard. $0 \mathrm{n}$ n'a jamais parlé directement de ma maladie. A près plusieurs années je vois plus par ses adions ce qu'il en pense, mais (... ). $0 \mathrm{n}$ aurait besoin d'un groupe de support dans la région pour les hommes dont les épouses sont malades et un autre, pour les femmes seulement. Tu sais, les hommes ont beaucoup de difficulté 
«P our les F ranco$M$ anitobaines, I' effet de la solidarité entre les consoeurs peut poser des problèmes, surtout lorsqu' on pense aux exigences qu' entraine la confidentialité, laquelle doit être respectée par tous les individus aptes à partiaper aux groupes de soutien... » à exprimer ce qu'ils ressentent au sujet du cancer du sein. Ils ne comprennent rien! I Is ne savent pas ce que ressent une femme qui a seulement un sein (... ).»

Pour les Franco-M anitobaines, l'effet de la solidarité entre les consoeurs peut poser des problèmes, surtout lorsqu'on pense aux exigences qu'entraîne la confidentialité, laquelle doit être respectée par tous les individus aptes à participer aux groupes de soutien; car, participer à un groupe de soutien implique la capacité de recevoir et de respecter les confidences de ses consoeurs, d'être instruites des desseins et des événements de l'expérience du cancer du sein et de ses conséquences dans la vie familiale des femmes franco-manitobaines. $0 \mathrm{r}$, un tel objectif n'est pas facile à atteindre lorsqu'on vit dans un village, un milieu où les francophones vivant en situation minoritaire se connaissent intimement et partagent aussi les activités communes. $\mathrm{C}$ e qui peut devenir difficile lorsqu'on a besoin de se sentir en confiance et parler de sa maladie sans toutefois briser l'intimité des vies humaines. D ifficile ne signifie pas impossible, à condition, bien sûr, de respecter les règles de la confidentialité.

«L es groupes de soutien sont importants pour venir en aide aux familles brisées et quelquefois détruites par la maladie $M$ ais il faut que cela soit confidentie!! $M$ ais je crois qu'il est possible de parler de soi-même dans des groupes sans que les gens en parlent aux autres Sinon, cela ne vaut pas la peine de se retrouver ensemble et de parler de soi en tout état de confiance II faut vraiment avoir confiance aux autres et exiger aussi de garder pour soi des histoires qui ne doivent pas devenir publiques.»

Avant même de recourir aux organisations dites formelles à la suite de l'annonce de la mauvaise nouvelle, la plupart des femmes rencontrées nous ont dit s'être tournées vers leurs proches, leur famille afin de recevoir un soutien et du courage pour lutter contre la maladie. C eux-ci représentent le système de réseautage à caractère informel et seront donc identifiés comme tels, à savoir 
«E n l'absence de la famille, les amies, les voisins et les personnes significatives qui parlent français dans leur communauté rurale peuvent souvent devenir un autre recours pour les femmes francomanitobaines» les réseaux familial, relationnel et naturel. La famille, c'est la parenté, au sens strict du terme (mari, mère, soeur, belle-soeur et cousine). O n remarquera que disposer de temps est une ressource nécessaire pour que la famille puisse venir jouer son rôle d'aide auprès des femmes vivant ou ayant vécu avec le cancer du sein.

«L orsque j' ai eu mon cancer du sein, l'aînée de mes filles $m$ 'a beaucoup aidée. E lle s' ocaupait de ses frères et de ses soeurs $L$ e soir, mon mari m'aidait aussi avec les enfants, 》

«M a cousine qui a eu elle-même le cancer du sein est venue $\mathrm{m}$ 'aider. $E$ lle $\mathrm{m}$ 'a beaucoup encouragée $C$ 'est aussi une très bonne amie. $\mathrm{N}$ ous sommes près l'une de l'autre»

À ce réseau familial se greffe le réseau relationnel où se retrouvent des amis, des voisins, des personnes significatives parce que fréquentées sur une base régulière. C ette forme d'aide n'est pas en soi un phénomène nouveau. En l'absence de la famille, les amies, les voisins et les personnes significatives qui parlent français dans leur communauté rurale peuvent souvent devenir un autre recours pour les femmes franco-manitobaines. $C$ 'est du moins ce qui ressort des entrevues.

"L es amies francophones que j'ai toujours eues sont venues me voir pour m'encourager lorsque j'ai eu le cancer du sein (... ). Les amies qui n'ont jamais eu le cancer du sein font ce qu'elles peuvent pour me comprendre (... ). L orsque tu perds un sein ou te rapproches de la mort, tu peux diffiàlement $t$ ' imaginer ce qu' une femme peut ressentir, à moins de l'avoir vécu toimême (... ).»

"J'ai beaucoup d'amies de femmes de la région qui ont eu le cancer du sein et une mastectomie pour contrôler leur tumeur. E lles m' ont beaucoup aidée et $m$ ' aident encore à traverser ma maladie. $E$ lles me comprennent et 
«0 $n$ attribue au prêtre du village des vertus et on lui donne un pouvoir d'influence relativement important dans la façon de vivre avec sa maladie et de composer spirituellement avec sa propre réalité. » c'est tout ce qui compte L orsqu' on se rencontre, on $s^{\prime}$ encourage l'une l'autre. $0 \mathrm{n}$ ne parle par vraiment de notre maladie parce qu' on a toutes vécu la même maladie (... ). M ais on se parle d'autres choses.»

$D$ ans les groupes de soutien, on retrouve également les personnes qui offrent de l'aide en période difficile. $D$ ans la plupart des cas, les Franco- $M$ anitobaines qui ont été traitées à l'H ôpital Général de Saint-Boniface ont fait état de la présence des religieux francophones. Ainsi donc, ces derniers viennent en aide à ces femmes qui vivent des temps difficiles et leur assurent un suivi par les pratiques religieuses. Le type d'aide qu'ils fournissent est apprécié et souhaité par les catholiques pratiquantes qui ont recours aux services des religieux.

«Pendant mon séjour à l'hôpital, une gentille religieuse francophone est venue passer les soirées avec moi (... ). Elle apportait avec elle son petit livre de prières E lle m'a aussi donné un de ces livres pour que je puisse l'utiliser à chaque jour de ma convalescence à I'hôpital. II y avait des passages de la Bible que j'utilisais pour m'encourager lorsque j'étais découragée ou que je pleurais seule dans ma petite chambre (... ). E n l'écoutant me parler, j'essayais de me convaincre que je me devais d'être forte L orsque mon mari était à nouveau près de moi, je redevenais une femme forte. L orsqu'il me quittait, je m'écroulais à nouveau. E t la religieuse venait s' asseoir près de moi... »

Puisil y a le prêtre francophone du village. En tant que confident et représentant de l'ordre religieux, il joue un rôle actif dans la vie quotidienne des femmes franco-manitobaines. Q uelquefois, il joue un rôle tampon, voire de consultant entre la Francomanitobaine et sa famille. $\mathrm{O}$ attribue au prêtre du village des vertus et on lui donne un pouvoir d'influence relativement important dans la façon de vivre avec sa maladie et de composer spirituellement avec sa propre réalité. O n croit en Dieu et en la communion des Saints. $0 \mathrm{n}$ achète des messes, on fait brûler des 
lampions. Voilà quelques pratiques culturelles et religieuses qui font partie intégrante de la vie quotidienne de ces femmes et qui permettent d'atténuer les conséquences psychologiques et sociales de leur état.

«L e prêtre (francophone) du village m'a beaucoup aidée à traverser la maladie. II venait me voir et $\mathrm{m}$ 'encourageait à continuer ma lutte (... ). Je crois en la communion des Saints. II priait pour moi. Tu saiş, tout cela m'aidait à mieux vivre avec mes problèmes, »

«L e prêtre (francophone) vient souvent à la maison et on prie ensemble. Je brûle des chandelles E t pendant quej'étais à l'hôpital, il en brûlait pour moi. II mel'a dit. II m'a aussi dit qu'il offrait des messes pour moi. Tu t'imagines! II a toujours été pour moi un confident.»

Enfin, les aidantes naturelles qui parlent français représentent pour les $F$ ranco- $M$ anitobaines une condition sine qua non à leur participation au groupe. II s'agit en fait de personnes de la communauté d'appartenance ethnique auxquelles on reconnaît une compétence particulière pour fournir de l'aide aux malades en période difficile. $D$ ans les témoignages, on remarque l'influence prépondérante des valeurs religieuses dans l'expérience de la maladie, ce qui permet aux patientes de se rapprocher davantage et de partager un vécu commun (médical et social).

«D es femmes du village sont venues m'aider. 0 ui, ce sont des francophones parce que moi je ne parle pas anglais. D eux d'entre elles avaient déjà eu le cancer du sein et l'autre était la religieuse qui m'enseignait des cours sur la Bible. Elles sont toutes trois venues pour m'encourager lorsque j'ai regu la nouvelle. E lles m'ont dit de m'accocher à la vie, de garder l'espoir. Elles sont beaucoup plus vieilles que moi (... ). L es années ont passé et elles sont toujours là à m' encourager. E lles m'ont beaucoup parléde prévention en allant à mes 
visites chez le médecin. Et moi, j'encourage à mon tour d'autres femmes de la région qui sont devenues des victimes du cancer du sein. Je parle aussi à mes filles de prévention. Pour mieux traverser mon expérience, il a fallu que j'aie la reconstruction du sein. $L$ a prothèse ne suffisait pas pour pallier à mon manque physique et accepter mon problème»

«D epuis mon expérience avec le cancer du sein, je consacre une grande partie de ma vie à aider les femmes francophones à vivre avec le cancer du sein. Tu sais, il n'y a pas beaucoup de francophones id qui peuvent aider les autres Je téléphone aux femmes francophones et les encourage dans leur expérience (... ). II $\mathrm{m}^{\prime}$ arrive aussi de les accompagner dans leurs traitements chimiothérapiques ou de radiation (... ). Parce que j'ai eu mon cancer du sein en 1969, je deviens un exemple pour les autres femmes. Je n' ai pas eu de dépression. À ce moment, je me suis inscrite à un cours qui nous permettait de devenir bénévole et de savoir comment parler aux femmes malades (... ). Je parle aux femmes de la foi et de l'espérance en $D$ ieu (... ). L a maladien'a rien à voir avec nos croyances religieuses mais elles peuvent nous aider à traverser cette expérience qui est d'abord physique (... ).»

Toutefois, ces groupes de soutien que constituent la famille, les amis, les voisins, les personnes qui offrent de l'aide en période difficile et les aidantes naturelles, et qui absorbent une proportion importante de problèmes psychologiques et sociaux ne se limitent pas au domaine domestique et familial mais débordent parfois les frontières géographiques et linguistiques. II arrive que des patientes bilingues participent à W innipeg à des rencontres sociales organisées par les groupes de soutien anglophones. La participation à ces rencontres exige de la part des femmes mobilité, autonomie et capacité physique. 
«C ette approche téléphonique et communautaire réalisée cette fois- a par des aidantes naturelles représente des initiatives positives, diverses et concrètes qui voient le jour dans la région de la $R$ ivière $R$ ouge depuis plusieurs années »
«A près avoir eu la mastectomie, j'ai participé à des rencontres sodales qui ont eu lieu à W innipeg. Tout se passait en anglais! Parce que je ne conduis pas, mon mari venait avec moi. J'avais du plaisir à être avec les femmes qui avaient eu aussi le cancer du sein. Le groupe organisait des parades de mode C ela m'encourageait beaucoup! $M$ ais pendant l'hiver et à cause des tempêtes de neige, cela nous empêchait d'aller à toutes les sessions »

Parallèlement à ces rencontres, il existe aussi d'autres groupes de soutien informelsqui répondent aux besoinsimmédiats desfemmes de la région. $0 \mathrm{n}$ pense ici à l'encouragement provenant de femmes vivant dans d'autres villages.

«'ai des amies francophones qui sont venues $m$ 'encourager. $\mathrm{C}$ es femmes ont elles aussi le cancer du sein. U ne d'entre elles vit dans un autre village situé dans la région. Elle m'a téléphoné pour me dire qu'elle avait eu le cancer du sein. E lle y a survéa. E lle m'a parlé des chances de survie E lle m'a aussi encouragéà lui téléphoner dans des moments difficiles, des périodes de découragement (... ).»

Cette approche téléphonique et communautaire réalisée cette fois-ci par des aidantes naturelles représente des initiatives positives, diverses et concrètes qui voient le jour dans la région de la $R$ ivière $R$ ouge depuis plusieurs années. Et ces initiatives sont organisées parce que les femmes franco-manitobaines veulent bien qu'elles existent. Ces femmes actives ont également une façon particulière de prendre leur vie en main et de se regrouper pour ainsi venir en aide aux autres femmes isolées. Précisons qu'elles ne le font en vertu d'aucune obligation contractuelle, mais parce qu'elles croient qu'il est possible de vivre avec le cancer du sein et de partager un univers d'événements à l'intérieur duquel les femmes se retrouvent. $U$ ne telle croyance s'explique en partie par les valeurs culturelles et religieuses, et par le sentiment social d'appartenir à un groupe relié à un ensemble de significations 
particulières. Ainsi, on dira que celles-ci se comprennent dans l'expérience de la maladie, telle qu'elle prend forme au coeur de la vie quotidienne des Franco- $M$ anitobaines, et ce, dans un contexte social marqué par l'avancement de la médecine moderne. Enfin, le fait de vivre dispersé en tant que sous-groupe et de vivre en tant que francophones en situation minoritaire a une influence sur la façon de vivre sa maladie et incite les femmes à organiser des groupes de soutien.

\section{Conclusion}

C es initiatives communautaires développées par et pour les femmes francophones veulent faciliter la prise en charge de leur santé. C es femmes se définissent par rapport à une communauté d'appartenance ethnique qui s'exprime par le sentiment de partager un héritage culturel distinct (la langue) des autres groupes ethniques, ce qui les rapproche davantage les unes des autres et les définit en tant que groupe de femmes franco-manitobaines. De plus, ces femmes vivant ou ayant vécu avec le cancer du sein ont le sentiment d'appartenir à un groupe particulier se distinguant de la communauté franco-manitobaine par son expérience de la maladie, avec les conséquences psychologiques, physiques et sociales qui s'y rattachent. II existe donc une identité propre à ce sous-groupe de femmes qui se développe dans les échanges avec leurs consoeurs francophones vivant des rapports similaires avec le social et le médical. C ette identification s'explique par la culture ou sous culture qui est au centre de leur expérience de la maladie, telle qu'elle s'enracine au coeur de leur quotidien. Et si les femmes font elles-mêmes référence à cette identification, c'est qu'elles reflètent le caractère distinctif et culturel de leur sous groupe de femmes franco-manitobaines aux prises ou ayant été aux prises avec la maladie.

Les différents groupes de soutien informellement organisés répondent à des besoins particuliers, tant au niveau individuel 
que collectif, et ces besoins reflètent le milieu dans lequel elles vivent. Les services offerts par des femmes francophones, par exemple, permettent l'amélioration de la condition des autres femmes survivant au cancer du sein et dont le corps handicapé est marqué par le savoir médical et ses techniques chirurgicales. Les femmes sont engagées avec le corps médical afin de combattre la maladie et ses ravages dans la vie des familles atteintes et des autres à venir. Elles sont également engagées à aider les autres femmes qui vivent ou ont vécu l'expérience de la maladie pour qu'elles puissent prendre en main leur santé physique, mentale, spirituelle et sociale.

Les propos de cet article visaient donc à contribuer à une meilleure connaissance de la réalité des femmes francomanitobaines vivant ou ayant vécu avec le cancer du sein. II s'agissait d'expliquer la complexité et les difficultés de vivre le quotidien et d'accepter la réalité de la maladie qui affecte aussi les individus qui entourent les femmes qui en sont atteintes. Évidemment, cette analyse est partielle quant à l'usage de sa méthode d'analyse du discours, mais son contenu est fort révélateur parce qu'il nous permet de mieux comprendre différents éléments d'importance pour la santé communautaire des femmes franco-manitobaines et le sens (ou la capacité) d'organisation dont elles nous ont fait part dans leurs propos.

\section{Bibliographie}

BÉGIN, M onique (1989). L a refonte des soins de santé destinés aux femmes, 0 ttawa, Institut canadien de recherches sur les femmes (ICR EF).

BERTAU X , D aniel (1976). H istoire de vie - ou récit de pratiques? M éthodologie de l'approche biographique en sociologie, Paris, R apport du CO R DES.

D ELVEC CHIO-GO O D, M ary-Jo (1993). «O ncologie et temps narratif», Santé et allure, vol. 9, no 1, 19-40.

D O RVILL, H enri et al. (1994). «'exclusion des personnes handicapées», dans Fernand Dumont, Simon Langlois et $Y$ ves $M$ artin, éd. T raité des problèmes sodaux, Q uébec, Institut de recherche sur la culture, 711-738.

FO RTIN , Andrée (1994). «La famille: premier et ultime recours», dans Fernand D umont, Simon Langlois et $Y$ ves $M$ artin, éd, T raité des problèmes sodaux, $Q$ uébec, Institut de recherche sur la culture, 947-962. 
FO U C AU LT, M ichel (1972). H istoire de la folie, Paris, Presses universitaires de France, Collection. GAU THIER , Josée (1995). «Y at-il une vie après la mort», L es diplômés, no 385, 8-9.

GO FFM AN , Erving (1975). Stigmate - L es visages sociaux des handicapés, Paris, É ditions de M inuit. GOO D, Byron et M ary-Jo DELVEC CHIO -GOO D (1982). «Toward a M eaning C entered A nalysis of Popular IIIness C ategories: Fright IIIness and H eart Distress», dans I ran A.J. M arsella et G.M.W hite, ed. C ultural C onceptions of $M$ ental $H$ ealth andT herapy, Boston, D. R eidel Publishing, 141-146.

GO O D, Byron et M ary-Jo DELV EC C H IO -GO O D (1981). «T he M eaning of Symptoms:A C ultural $\mathrm{H}$ ermeneutic of $\mathrm{C}$ linical Practice», dans Leon E isenberg andArthur K leinman, ed. T he R elevance of Sodial Science for M edicine, Boston, D. R eidel Publishing, 165-197.

GR ELL, Paul (1986). «Les récits de vie: une méthologie pour dépasser les réalités partielles», dans $D$ anielle D esmarais et Paul Grell, éd. L es réits de vie, M ontréal, Éditions Saint- $M$ artin, 35-51.

HER ZLIC H , C laudine (1984). Santé et maladie. A nalyse d'une représentation sodale, Paris, Éditions de M inuit.

H O U LE, Gilles (1986). «H istoires et récits de vie: la redécouverte obligée du sens commun», dans $D$ anielle D esmarais et Paul G rell, éd. L es réats de vie, M ontréal, Éditions Saint- M artin, 35-51.

KLEIN M AN ,Arthur (1986). «Social $O$ rigins of D istress and D isease: D epression N eurasthenia and Pain in M odern C hina», C urrent A nthropology, vol. 27, no 5, 499-509.

LE GALL, Didier (1987). «es récits de vie: approcher le social par la pratique», dans Jean-Pierre D eslauriers, éd. L es méthodes de la recherche qualitative, Sillery, Presses de I'U niversité du Q uébec, 35-48.

SAILLAN T, Francine (1988). C ulture et cancer, M ontréal, É ditions Saint-M artin.

SC H U T Z, Alfred (1987). Le chercheur et le quotidien, Paris, M éridiens K lincksieck.

ST AT IST IQ U E CAN ADA (1995). C anadian C ancer Statistics, $\mathrm{O}$ ttawa, $\mathrm{N}$ ational C ancer Institute of Canada.

TO R R IE,A . (1971). « Like a Bird with a Broken W ing», W orld M ediane, Avril.

W O N G, Agnes (1995). «T he Inhumanity of Fairness: R ationing R esources for R econstructive B reast Surgery», C anadian M edical A ssodation Journal, vol. 152, no 4, 577-579. 